REGARDS

SUR L'ECONOMIE ALLEMANDE

BULLETIN ECONOMIQUE DU CIRAC
Regards sur l'économie allemande

Bulletin économique du CIRAC

112 | 2014

Varia

\title{
Banque centrale européenne : monnaie, justice, démocratie - de Karlsruhe à Luxembourg -
}

\section{Peter-Christian Müller-Graff}

Traducteur : Isabelle Bourgeois

\section{OpenEdition}

\section{Journals}

Édition électronique

URL : http://journals.openedition.org/rea/4657

DOI : $10.4000 /$ rea. 4657

ISBN : 978-2-8218-0826-3

ISSN : 1965-0787

Éditeur

CIRAC

Édition imprimée

Date de publication : 28 avril 2014

Pagination : 17-24

ISSN : 1156-8992

\section{Référence électronique}

Peter-Christian Müller-Graff, « Banque centrale européenne : monnaie, justice, démocratie - de

Karlsruhe à Luxembourg - », Regards sur l'économie allemande [En ligne], 112 | avril 2014, mis en ligne le 01 avril 2016, consulté le 01 mai 2019. URL : http://journals.openedition.org/rea/4657 ; DOI : 10.4000/ rea.4657 


\section{Banque centrale européenne : monnaie, justice, démocratie - de Karlsruhe à Luxembourg -}

\section{Peter-Christian Müller-Graff}

La Banque centrale européenne (BCE) passe en justice... D'abord devant le Tribunal constitutionnel fédéral de Karlsruhe (TCF) et maintenant, depuis le 7 février 2014, aussi devant la Cour européenne de justice (CJUE) de Luxembourg.

A cette date, la Cour de Karlsruhe avait en effet décidé à six voix contre deux de renvoyer devant la CJUE le jugement de fond d'un point de l'affaire dont elle avait été saisie entre autres à propos du Mécanisme européen de stabilité (MES) en 2012 (voir REA 106/2012), à savoir la question de la conformité au droit communautaire du programme de rachat d'obligations émises par les Etats en crise de la zone Euro, dénommé "Outright Monetary Transactions " (OMT), et annoncé par la BCE le 6 septembre 2012. Si ce renvoi à l'échelon supérieur est une première dans l'histoire de I'UE, il n'en était pas moins prévisible...

\section{Le programme OMT et le droit de vote}

La Cour de Karlsruhe avait été saisie en 2012 notamment par le groupe parlementaire Bündnis 90/Die Grünen et, particulièrement important dans ce cas précis, par de nombreux citoyens allemands qui ont fait là usage du droit de saisine directe que leur confie la Constitution dès lors qu'ils estiment qu'un de leurs droits fondamentaux est lésé par le législateur (plainte en constitutionnalité) : en l'occurrence par le comportement de la BCE et des organes de l'Etat allemand dans la gestion de la crise de la dette souveraine de certains Etats de la zone Euro. Ils incriminent la BCE d'avoir enfreint la Constitution en violant leur droit fondamental à participer au fonctionnement démocratique de la République fédérale, incarné par le droit de vote (art. 38 de la Loi fondamentale, LF).

Le Tribunal constitutionnel fédéral considère une telle saisine comme légitime puisqu'il considère que tout citoyen allemand est habilité, en qualité d'acteur du principe de démocratie, à saisir cette cour suprême dès lors qu'il affirme de manière substantielle que son droit de vote au Bundestag est vidé de son sens BVerfGE 89, 155, 171 ; BVerfGE 123, 267, 330 ; BVerfGE 129, 124, 168). En matière de politique européenne, cette violation s'effectue ou bien par un transfert de compétences de la RFA à l'échelon de l'UE qui affaiblit les pouvoirs du Bundestag dans une ampleur contraire à la Constitution (BVerfGE 123, 267, $330,351 \mathrm{sq}$.) ou bien lorsque l'UE agit en outrepassant les compétences que lui a conférées le Bundestag via les traités ("ultra vires »). C'est ce dernier point qui constitue le motif de la saisine à propos du programme OMT de la BCE. Quant au fond, le TCF émet lui aussi des doutes quant à la conformité de ce programme avec le droit primaire de l'UE tel qu'il a été ratifié par la RFA.

Quel est donc ce lien, qui peut sembler étrange aux yeux d'un lecteur français, entre le programme OMT et les droits fondamentaux des plaignants, sachant que ces derniers considèrent que la BCE viole leur droit de vote? Avant d'y revenir de manière plus détaillée, voyons dans le détail l'exposé des motifs des

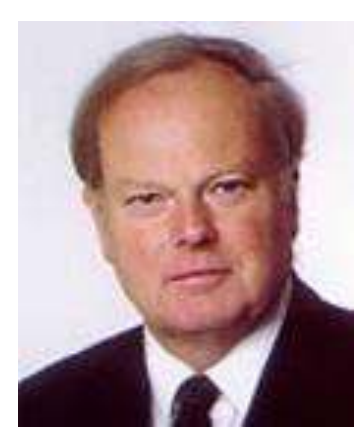

Prof. P.-C. Müller-Graff, Directeur de 1'Institut für deutsches und europäisches Gesellschafts- und Wirtschaftsrecht, Université de Heidelberg

La Cour de Karlsruhe avait été saisie par des citoyens allemands

Motif : violation du droit de vote par la BCE... 
... et son programme OMT

La BCE aurait outrepassé ses compétences

Renvoi devant la CJUE plaignants. Selon eux, en adoptant le programme OMT, la BCE outrepasserait les compétences limitées que lui a conférées le peuple allemand via l'acte démocratique qu'est la Loi d'approbation du Traité de Maastricht adoptée par le Bundestag et le Bundesrat.

Dit en termes plus juridiques : premièrement, par cette politique d'achat d'obligations d'Etat, la BCE n'accomplirait pas sa mission de politique monétaire (au sens de l'art. 127 du Traité sur le fonctionnement de l'UE, TFUE), mais mènerait une politique économique et une politique européenne, toutes deux étrangères à ses missions. Deuxièmement, la BCE violerait l'interdiction de monétariser la dette et l'interdiction qui lui est faite expressément de contourner cette disposition par l'acquisition directe d'obligations d'Etat sur le marché primaire (art. 123 TFUE). Le programme OMT équivaudrait donc à un contournement de cette interdiction.

Dans le fond de l'affaire dont avait à connaître la Cour de Karlsruhe, et que cette dernière a renvoyé à la CJUE, il s'agit donc d'abord d'un contrôle en conformité avec le droit communautaire de cette décision du Conseil des gouverneurs de la BCE et ensuite, quant au fond, de l'interprétation à donner aux dispositions relatives aux compétences respectives contenues dans le droit primaire de l'UE tel qu'il a été construit par les Etats membres.

Or, dès lors qu'une juridiction nationale, subsidiaire, est saisie d'une plainte en la matière, l'instance chargée de cette interprétation au sein de la " communauté de droit » (Walter Hallstein) qu'est l'UE est en dernier ressort la Cour européenne de justice. Voilà pourquoi, et conformément à cette articulation, le Tribunal constitutionnel fédéral, juridiction suprême à l'échelon national de l'Allemagne, a renvoyé cette question de fond à la CJUE, seule habilitée à statuer en dernière instance (art. $267 \S 1 \mathrm{~b}$ et art. 3 TFUE). Ce faisant, le TCF part du présupposé que la décision du Conseil des gouverneurs de la BCE a qualité d'acte d'un organe de l'UE au sens de l'art. 267 §1 B du TFUE.

Ensuite, lorsque la CJUE aura statué à l'échelon communautaire, c'est au TCF qu'il reviendra en dernière instance d'interpréter, à l'échelon national cette fois, la portée de l'arrêt de la CJUE en ce qui concerne la plainte dont il avait été saisi en Allemagne. De quelles questions s'agit-il concrètement $(A)$ ? Comment les considère la Cour de Karlsruhe $(B)$ ? Comment pourrait les aborder la Cour de Luxembourg (C) ? Et quelle est l'articulation entre la BCE, le droit, la jurisprudence et la démocratie (D) ?

\section{A/ Les questions soumises par le TCF à la CJUE}

Le TCF a soumis à la CJUE pour décision préjudicielle deux questions à la fois précises et détaillées : une question centrale en deux points et une question accessoire, elle aussi en deux points.

La question centrale est celle de savoir si les mesures adoptées par la BCE (elles sont présentées de manière circonstanciée) sont incompatibles avec certaines dispositions du droit communautaire. La question accessoire n'a été posée que pour le cas où la CJUE considérerait que la décision sur le programme OMT prise par le conseil de la BCE ne constitue pas un objet valable d'une procédure de renvoi auprès de la CJUE selon l'art. 267 \& 1 b TFUE. Et pour prévenir ce cas, la Cour de Karlsruhe a dès lors formulé cette question en inversant en quelque sorte les termes de sa question centrale sous cette forme : le droit communautaire autorise-t-il la zone Euro de mettre en œuvre les mesures présentées de manière circonstanciée dans la question centrale?

Une question centrale en deux points : ...

Voyons plus précisément la question centrale. Elle se divise en deux points : d'une part, celui de savoir si, en décidant le programme OMT, la BCE a outrepassé ses compétences et plus précisément si celui-ci est compatible avec les 
art. 119, $127 \S 1$ et 2 du TFUE ; d'autre part, celui de savoir si ce programme viole l'interdiction de monétariser la dette (art. 213 TFUE). A première vue, ces deux points semblent purement techniques, mais en réalité, ils cherchent à apporter une réponse claire à une question hautement politique : quelles sont les possibilités ouvertes à l'action de la BCE et quelles sont leurs limites dans la crise de la dette souveraine que rencontrent certains Etats de la zone Euro ? Sachant que, en tout état de cause, la BCE est tenue de respecter les dispositions afférentes du Traité sur le fonctionnement de l'UE, et tout particulièrement ses art. $119 \mathrm{sq.}$

Sur le premier point, le TCF formule la question des compétences outrepassées de la BCE selon quatre perspectives : d'abord au regard de la conditionnalité de l'articulation du programme OMT avec les programmes d'aide économique du Fonds européen de stabilité financière (FESF) ou du Mécanisme européen de stabilité (MES) (conditionnalité) ; puis, au regard de la limitation prévue de l'achat d'obligations à celle de certains Etats seulement (sélectivité) ; ensuite, sous l'angle de l'achat prévu d'obligations des Etats en difficulté en complément des programmes d'aide des Etats de la zone Euro, à savoir le FESF et le MES (parallélisme) ; enfin, sous celui du risque que le programme OMT permette de contourner les limitations et les conditions liées à la mise en œuvre des mécanismes FESF et MES (risque de contournement).

Quant au deuxième point (la violation de l'interdiction de monétariser la dette), le TCF l'aborde sous cinq aspects de l'accord sur le programme OMT : premièrement sous celui de l'absence d'une limitation quantitative de l'achat d'obligations d'Etat (volume); deuxièmement, sous celui de l'absence de délai entre l'émission d'obligations d'Etat sur le marché primaire (c'est-à-dire leur achat direct à l'Etat en question, sans intermédiaire, par des investisseurs non étatiques) et leur acquisition par le Système européen des banques centrales (SEBC) sur le marché secondaire, c'est-à-dire leur rachat effectué ensuite aux investisseurs non étatiques (formation des prix des obligations). Le troisième aspect concerne la possibilité de détenir jusqu'à la date d'échéance la totalité des obligations d'Etat rachetées (intervention dans la logique du marché) ; le quatrième est celui de l'absence d'exigences spécifiques concernant la solidité des obligations d'Etat à acquérir (risque de défaillance) ; enfin, le cinquième soulève la question de l'égalité de traitement entre le SEBC et les détenteurs privés et autres des obligations d'Etat en cas de restructuration de la dette (« hair cut »).

Dans la question accessoire soumise à la CJUE, la Cour de Karlsruhe thématise les aspects déclinés dans sa question centrale en les inversant pour adopter la perspective des compétences positives de la BCE. Le TCF demande ainsi si le droit communautaire autorise l'Eurosystème (qui réunit les banques centrales de tous les Etats de l'UE, qu'ils soient membres de la zone Euro ou non) de mettre en œuvre les quatre mesures décrites dans la première partie de la question centrale. Elle demande également si la compétence de l'Eurosystème en ce qui concerne l'achat et la vente de titres sur le marché interbancaire et le pilotage des taux d'intérêt (opérations d'open market) l'autorise à racheter sur le marché primaire à l'investisseur acquéreur des obligations d'Etat nouvellement émises - et ce, premièrement sans limitation de volume, deuxièmement sans tenir compte d'un délai par rapport à l'achat de ces obligations nouvellement émises par le premier investisseur, troisièmement l'autorise à les détenir jusqu'à la date d'échéance, quatrièmement à renoncer aux exigences de solvabilité et ce, cinquièmement, dans l'optique de l'égalité de traitement avec les autres détenteurs dans le cas d'une annulation de la dette.

La Cour de Karlsruhe ajoute à cela une sixième question qui est de savoir si le droit communautaire permet à l'Eurosystème « d'influer sur la formation des prix des obligations par l'annonce d'intentions de rachat ou par toute autre mesure concomitante avec l'émission de nouvelles obligations par les Etats membres de la zone Euro (incitation à l'acquisition de nouvelles obligations) ».
... compétences outrepassées ?

... violation de l'interdiction de monétariser la dette?

Une question accessoire sur l'articulation BCE/Eurosystème 
Quel lien avec le droit de vote et le principe de démocratie?
Elle se réfère là à l'intention de la BCE d'annuler le risque, pour les acquéreurs de nouvelles obligations émises par les Etats en crise, de se trouver dans l'impossibilité de les revendre et de supporter les pertes induites.

Au vu de ces questions relatives au droit communautaire, il est difficile de saisir immédiatement ce qu'elles ont à voir avec le droit de vote des citoyens allemands aux élections au Bundestag et avec le principe de démocratie. Certes, ce lien ne fait pas non plus l'unanimité parmi les juges de Karlsruhe, et deux d'entre eux ont exposé une vue divergente dans les considérants de la décision de renvoi à la CJUE, allant jusqu'à estimer que la saisine du TCF était dès lors infondée (Lübbe-Wolff, points 13 sq., et Gerhard, points 5 sq. et 8 sq.).

Si ce lien ne s'impose pas à l'évidence, il devient néanmoins parfaitement intelligible quand on se penche sur l'argumentation du Tribunal constitutionnel fédéral. En effet, si on considère premièrement que le droit de vote ne se réduit pas au droit des citoyens de déposer un bulletin dans l'urne, mais qu'il inclut également le droit à la participation active à la définition démocratique de la substance même des conditions de vie en Allemagne, que deuxièmement, le Bundestag a renoncé, dans les limites d'un cadre défini dans le respect du droit, à ce droit de définition en matière de politique monétaire au bénéfice de l'institution indépendante qu'est la BCE, le droit de vote se trouve violé, estime la Cour, si troisièmement, la BCE s'arroge des droits au-delà de la mission qui lui a été confiée.

\section{Le rôle clé du principe de démocratie et du droit de vote}

Le principe de démocratie est au fondement de l'intégration de l'Allemagne dans l'UE.

Celle-ci est inscrite dans la Constitution.

Dans le préambule de la Loi fondamentale, d'abord : “Conscient de sa responsabilité devant Dieu et devant les hommes, animé de la volonté de servir la paix du monde en qualité de membre égal en droits dans une Europe unie, le peuple allemand s'est donné la présente Loi fondamentale en vertu de son pouvoir constituant ».

Et dans l'art. 23, § 1 de la Loi fondamentale, qui pose le principe de «l'ouverture de l'Etat » allemand (offene Staatlichkeit) à son intégration dans l'UE : "Pour l'édification d'une Europe unie, la République fédérale d'Allemagne concourt au développement de I'Union européenne qui est attachée aux principes fédératifs, sociaux, d'Etat de droit et de démocratie ainsi qu'au principe de subsidiarité et qui garantit une protection des droits fondamentaux substantiellement comparable à celle de la présente Loi fondamentale. A cet effet, la Fédération peut transférer des droits de souveraineté par une loi approuvée par le Bundesrat. L'article 79, al. 2 et 3 est applicable à l'institution de I'Union européenne ainsi qu'aux modifications de ses bases conventionnelles et aux autres textes comparables qui modifient ou complètent la présente Loi fondamentale dans son contenu ou rendent possibles de tels compléments ou modifications ".

L'art. 79 de la Loi fondamentale dispose que, si les principes fondateurs de la Loi fondamentales sont immuables en ce qui concerne l'Allemagne (dignité humaine, Etat de droit, fédéralisme, principes constitutifs de la structure de l'Etat), la Constitution allemande est ouverte à des modifications ultérieures dans le cadre de l'intégration européenne. La Cour de Karlsruhe, dans son arrêt rendu le 12-09-2012 à propos du MES, l'explicite ainsi : " l'art. 79, § 3 de la Loi fondamentale... ne (garantit) pas le caractère immuable du droit en l'état, mais la pérennité des structures et procédures qui assurent la vitalité du processus démocratique et qui, ce faisant, garantissent également la responsabilité budgétaire globale du parlement » (al. 222 de l'arrêt ; voir REA 106/2012). Mais ces modifications doivent se faire dans le respect du droit de vote. Celui-ci (art. 38 de la Loi fondamentale) incarne en effet l'essence même des principes démocratiques. Dès lors que la République fédérale contribue à l'œuvre européenne, ils s'appliquent aussi à l'UE. Dans son arrêt rendu le 30-06-2009 sur le Traité de Lisbonne (voir REA 92/2009), le TCF en déduit «le droit à l'autodétermination démocratique, à une participation libre et égale au pouvoir étatique exercé en Allemagne, ainsi qu'à l'observation du précepte démocratique, y inclus le respect du pouvoir constitutionnel du peuple » (al. 208). Dans son arrêt rendu le 07-09-2011 sur les aides budgétaires dans la zone Euro (voir REA 102/2011), le TCF avait exposé ainsi l'articulation entre l'échelon national et communautaire : « le droit du citoyen à la démocratie, qui repose foncièrement sur la dignité de l'Homme, ... se trouverait invalidé si le parlement abandonnait des éléments essentiels de l'autodétermination politique et retirait ainsi durablement au peuple son pouvoir d'influence démocratique ".

Du fait de l'intégration active de l'Allemagne dans I'UE et de ses implications pour les citoyens allemands, le principe d'autodétermination démocratique trouve donc également son application en droit communautaire.

(I.B. ; d'après : Müller-Graff, REA 92/2009, 102/2011 et 106/2012)

C'est le peuple souverain qui a défini le mandat de la BCE
Car les organes de l'Union européenne, donc la BCE aussi, n'ont le pouvoir d'exercer leur politique que conformément aux compétences confiées par les traités conclus par les Etats membres (principe de la subsidiarité et des limites de la compétence assignée, art. 2 \& 2 Traité de l'UE). Ils ne peuvent donc agir que dans le strict respect des règles en vigueur dans la communauté de droit européenne et qui ont été validées par le peuple souverain de chacun des Etats membres (Bundestag et Bundesrat en Allemagne). Dans le cas contraire, ils outrepassent leurs compétences, c'est-à-dire qu'ils agissent « ultra vires ». Dès 
lors, si on veut confier à la BCE des compétences dépassant cet acquis, il faut impérativement que les Etats membres signent en conséquence un nouveau traité. Celui-ci doit être adopté par le peuple souverain de chaque Etat membre et être conforme à sa Constitution. Voilà pourquoi les questions que soumet le TCF à la CJUE conjuguent le principe national de démocratie avec les limites assignées à l'action légitime de la BCE.

\section{B/ La position de la Cour de Karlsruhe}

Conformément à la tradition de la jurisprudence allemande, le Tribunal constitutionnel fédéral appuie sa décision sur une argumentation substantielle. II en conclut que le programme OMT est incompatible avec le droit communautaire (sous réserve de l'avis contraire de la CJUE ; point 55 de la décision), dès lors qu'aucune interprétation conforme n'est possible (points 99-100). Voici les principales lignes de son argumentation.

Pour la Cour de Karlsruhe, la BCE outrepasse son mandat en matière de politique monétaire en ce sens que, dans le système du droit de l'UE, le programme OMT adopté ne relève pas en premier lieu du champ de la politique monétaire, mais de celui de la politique économique; or la BCE n'a pas légitimité à mener des actions de politique économique (points 56-83). Le TCF parvient à cette conclusion selon quatre perspectives.

Dans un premier temps, la Cour considère l'objectif immédiat de l'adoption du programme OMT, à savoir l'intention de neutraliser la hausse des taux d'intérêt sur les obligations d'Etat imposée par des marchés sceptiques quant à la réalité de la discipline budgétaire de certains Etats de la zone Euro. Or cette neutralisation réduirait à néant la pression exercée par les marchés sur ces Etats pour les amener à respecter la discipline budgétaire et leur engagement responsable (point 71). II s'agit là d'un état de fait indéniable. Or, selon le Traité TFUE (art. 126) et la jurisprudence de la CJUE à propos du MES (arrêt Rs. C-370/12 du 27-11-2012 à propos de la conformité du Traité MES au droit communautaire ; " arrêt Pringle »; points 56-60 et 97, voir Müller-Graff, 2013), les aides budgétaires n'entrent pas dans le champ de la politique monétaire, mais dans celui de la politique économique, et celle-ci relève de la souveraineté des Etats membres (points 64-66 et 71 de la décision de Karlsruhe). Le traité TFUE ne prête à aucune équivoque. Par ailleurs, la préservation de l'intégrité de la zone Euro dans son périmètre actuel "n'est à l'évidence pas une mission de la politique monétaire", mais entre dans le domaine de compétence des Etats membres : la politique économique (point 72). Nul doute sur ce point. Rien ne permet non plus d'étayer l'approche selon laquelle l'objectif de " l'intégrité » de la zone Euro, autrement dit son périmètre et sa configuration, se fonderait sur celui de la stabilité des prix ; il pourrait tout au plus se fonder sur le fait que l'intervention de la BCE vise à soutenir globalement la politique économique au sein de l'UE (art. 127 § 1 TFUE).

Dans un deuxième temps, le TCF conclut à l'absence d'objectif de politique monétaire du programme OMT en s'appuyant sur son intention d'acquérir des obligations d'Etat de manière ciblée et sélective, et ce en franche contradiction avec les principes directeurs dont s'est dotée la BCE (point 73). Car foncièrement, ces derniers n'autorisent aucune différenciation. Reste à savoir si ces principes directeurs reflètent fidèlement le droit primaire de l'UE.

Dans un troisième temps, le TCF met en doute la qualité d'instrument de politique monétaire du programme OMT en s'appuyant sur le fait qu'il est soumis aux conditions d'attribution des programmes d'aide du MES qui, elles, relèvent de la politique économique (points 74-78). Ce doute ne se laisserait écarter que s'il s'agissait d'une mesure de soutien recevable menée dans le cadre de la politique économique générale au sein de l'UE. Enfin, le TCF met en lumière à juste titre la possibilité qu'offre le programme OMT de contourner les règles strictes de la conditionnalité des programmes d'aides du MES (point 79) et de
La BCE n'est pas habilitée à mener une politique économique

L'OMT risque de diluer la discipline budgétaire, ...

... entre en contradiction avec les principes directeurs de la BCE, ...

... permet de contourner la conditionnalité du MES... 
... et viole l'interdiction de monétariser la dette

L'OMT n'est conforme au droi que si lui sont posées des limites contrecarrer d'autant plus facilement leur fonction en matière de politique économique que le volume d'achat d'obligations n'est pas limité (points 80-83). Cette considération est lourde de conséquences.

La Cour de Karlsruhe parvient également à la conclusion que le programme OMT viole l'interdiction de monétariser la dette. Certes, il ne vise que l'achat d'obligations d'Etat sur le marché secondaire et, sous cet angle, ne viole nullement les termes de l'interdiction inscrite à l'art. $123 \S 1$ TFUE. Celui-ci interdit à la BCE et aux banques centrales l'acquisition "directe » d'obligations émises par les Etats membres. Or le TCF voit dans le mode de fonctionnement du programme OMT une manière illicite de contourner la teneur de l'art. 123 TFUE (points 85-94). II parvient à cette conclusion - conséquence logique de sa lecture des limites posées par le droit communautaire à la politique monétaire de la BCE - en considérant le seul objectif de ce programme, qui est de neutraliser la hausse des taux d'intérêt (voir plus haut), et son parallélisme avec les programmes d'aide du MES (idem).

A l'appui de cette thèse du contournement, il évoque des indices supplémentaires : la disposition à coopérer à l'avenir lors d'une restructuration de la dette concernant les obligations acquises ; le risque accru de la survenue d'un tel hair cut; la possibilité de détenir les obligations jusqu'à leur échéance ; et enfin l'intervention dans la formation des prix sur le marché obligataire que permet l'acquisition d'un volume important d'obligations d'Etat sur le marché secondaire, dans un laps de temps restreint après leur émission. Ces aspects pèsent lourd, car il apparaît à l'évidence que l'annonce de l'adoption d'un programme comme l'OMT incite les investisseurs privés à l'achat de nouvelles obligations dans la mesure où la BCE laisse ainsi entendre qu'elle est prête à couvrir le risque financier qu'ils encourent en se portant acquéreur.

Les points soulevés amènent le TCF à en conclure que le programme OMT ne peut être conforme au droit communautaire que s'il subit une interprétation conforme et qu'il s'avère possible de le limiter en volume et dans le temps (points 99-100). Et la cour énumère plusieurs limites qu'elle considère indispensables. Ainsi, le programme OMT ne doit pas permettre de contourner la conditionnalité des aides du MES, et il ne peut que soutenir la politique économique communautaire, mais pas s'y substituer. En ce qui concerne l'interdiction de monétariser la dette, premièrement, la contribution du programme OMT à un hair cut doit être exclue, deuxièmement, le volume des obligations qu'il lui est possible d'acquérir doit être plafonné et, troisièmement, toute intervention dans le processus de fixation des prix des obligations d'Etat doit être évitée dans la mesure du possible.

Voilà les interrogations déterminantes que vient de soumettre la Cour de Karlsruhe à celle de Luxembourg. C'est à elle qu'il revient maintenant de trancher, conformément aux missions qui lui sont imparties, et de dire le droit en cette matière hautement sensible. Sa réponse est attendue avec fébrilité...

\section{C/ Spéculations sur les réponses de la CJUE}

Etant donné la nouveauté de la problématique soulevée, seules des spéculations sur la jurisprudence à venir de la Cour de Luxembourg sont possibles.

Un défi historique

La seule certitude est que la CJUE se trouve confrontée à des doutes solides et substantiellement étayés sur la conformité avec le droit du programme OMT qu'a décidé de lancer la BCE. II ne lui sera pas possible d'échapper totalement à son devoir de statuer sur ces questions hautement sensibles en décidant de considérer ce programme comme un objet susceptible d'un recours devant sa juridiction (au sens de l'art. 267 TFUE). Car les aspects qu'il soulève sont réitérés par le TCF dans ses questions accessoires, substantiellement développées, sur les compétences positives de l'Eurosystème. Au vu de la portée historique de ce premier renvoi du TCF vers la CJUE, et de la problématique primordiale 
ainsi abordée, la CJUE serait bien inspirée de relever le défi d'une discussion sur le fond.

Cela est d'autant plus important que la portée des interrogations de la Cour de Karlsruhe va bien au-delà de la dimension économique et politique de l'UEM comme de l'UE dans son ensemble. Elle pose en effet avant tout la question de l'autorité du droit de I'Union comme celle du droit dans l'UE. Or, depuis l'adoption des mesures de stabilisation de l'UEM à partir du mois de mai 2010, cette autorité est vivement mise en doute dans l'espace public (voir Müller-Graff, 2012). Car l'octroi d'aides budgétaires transnationales a sérieusement mis à mal le principe au fondement de l'Union économique et monétaire, à savoir la souveraineté et la responsabilité de chaque Etat membre en ce qui concerne sa politique économique et budgétaire (voir REA 102/2011 et 106/2012).

Cela ne signifie toutefois pas que soit enfreint de manière générale le droit communautaire. C'est ce qu'a clairement démontré la CJUE dans son "arrêt Pringle » rendu à propos de la conformité avec l'art. 125 TFUE (indépendamment de la clarification apportée par l'art. $136 \S 3$ TFUE) de l'adoption du MES par les Etats membres - rappelant que cette conformité n'est valable que si sont remplies certaines conditions de nature à préserver le principe fondateur de I'UEM. Mais ces considérations ne laissent en rien augurer du respect ou non du droit communautaire par la BCE lorsqu'elle adopte le programme OMT ou plus généralement dans les réponses qu'elle apporte à la crise de la dette souveraine de certains Etats de la zone Euro.

Etant donné l'ampleur des doutes suscités par les mesures de sauvetage quant à l'autorité du droit de l'UE et à la fiabilité de la communauté de droit européenne, on ne peut que se réjouir à l'idée que la CJUE ait désormais la chance de clarifier la situation et de dire le droit sur ce programme OMT et la politique de la BCE tant controversés. Quant aux règles du jeu de la communauté de droit européenne, elles exigent que tous respectent l'arrêt à venir de la CJUE et se plient à sa jurisprudence - de la Banque centrale européenne au Tribunal constitutionnel fédéral.

Certes, toute prévision concernant la manière dont une juridiction va interpréter une disposition légale relève de la pure spéculation. Mais en même temps, tout tribunal est tenu de se mouvoir à l'intérieur du cadre fixé par le législateur démocratiquement légitimé dans cette fonction. Dans le cas présent, la CJUE se meut donc dans le champ des traités ratifiés par les Etats membres de l'UE. Elle est tenue pareillement de se mouvoir à l'intérieur du cadre des quatre méthodologies d'interprétation officiellement reconnues, autrement dit le droit canon que constituent les méthodes grammaticale, systématique, historique et téléologique. Or dans le droit de l'Union, plurilingue par définition, l'orientation par rapport à la raison d'être d'une norme est déterminante. Elle l'est tout particulièrement dans le cas présent où la CJUE a à interpréter l'interdiction de la monétarisation de la dette. En outre, pour respecter les principes de la sécurité du droit et de l'égalité devant le droit, la CJUE doit se conformer à sa propre logique. En l'occurrence, cela signifie notamment que la CJUE doit éviter que son interprétation entre en contradiction avec les considérants sur la distinction entre politique économique et politique monétaire tels qu'ils figurent dans "l'arrêt Pringle » rendu à propos du MES.

Pour conclure, on peut donc s'attendre à ce que la Cour de Luxembourg reprenne à son compte la suggestion faite par celle de Karslruhe, à savoir de donner du programme OMT une interprétation en conformité avec le droit communautaire ou, si ce n'est pas possible, d'en réduire la portée, voire d'en invalider certaines dispositions. La tâche n'est certes pas aisée, mais la CJUE est désormais investie de la mission de la mener à bien en faisant preuve d'intelligence. Certes, elle peut toujours être tentée de ne pas se prononcer en renvoyant dans sa décision à la latitude d'interprétation laissée à l'appréciation du politique ; il ne lui sera néanmoins guère loisible de céder à cette tentation,
Les mesures de sauvetage ont mis à mal la souveraineté des Etats

L'autorité du droit communautaire en question

L'interprétation de la CJUE est strictement encadrée

Une réponse dilatoire est impossible 
pour une raison au moins. La BCE ne peut en effet user de sa latitude d'action que parce que, de par son statut, elle agit en toute indépendance des procédures politiques en vigueur dans nos démocraties. Mais elle ne peut ni définir le périmètre de cette indépendance statutaire ni encore moins l'élargir de sa propre initiative, car ce périmètre et ses missions ont été définis par les Etats membres grâce à des processus de ratification démocratiques, et la Cour de Luxembourg est pour sa part chargée d'interpréter les différentes dispositions ainsi adoptées. Seule une modification des traités permettrait d'accroître les missions et la latitude d'action politique de la BCE.

C'EST BIEN DANS CE TRIANGLE BCE - DROIT/JUSTICE - DÉMOCRATIE que se situe la problématique. D'abord, parce que la $\mathrm{BCE}$, en sa qualité d'organe supranational, a été dotée de l'indépendance vis-à-vis des procédures politiques et démocratiques des Etats membres et de l'UE pour garantir la préservation de la stabilité des prix (art. 127 TFUE) - cette indépendance s'impose à l'évidence (voir von Arnim, 1977) - grâce à la ratification de ce statut d'exception par le peuple souverain de tous les Etats membres. Ensuite parce que, justement à cause de cette 'carte blanche' qui lui a été démocratiquement confiée, l'action de la BCE ne peut s'effectuer que dans les limites imposées par le fondement juridique, donc les textes qui définissent son statut particulier tout en en fixant les limites. Si cela n'était pas le cas, les Etats membres verraient leur souveraineté politique réduite bien au-delà de la part qu'ils ont librement accepté de céder à la BCE par la voie des traités. Enfin, parce que le respect des limites ainsi fixées doit pouvoir être soumis au contrôle de la juridiction lorsque survient un litige sur la question de savoir si la BCE agit conformément aux compétences qui lui ont été confiées ou si sa politique les dépasse. Faute d'un tel contrôle, aucune règle de droit ne serait applicable, avec pour conséquence le risque de voir la BCE s'arroger arbitrairement des pouvoirs.

Voilà pourquoi il revient à la juridiction de tirer au clair, à propos du programme OMT, la question de savoir comment s'articule en droit communautaire le lien, établi conjointement par les Etats membres de l'UE, entre la Banque centrale européenne et la démocratie, c'est-à-dire le peuple souverain. Voilà pourquoi la $\mathrm{BCE}$ passe en justice. C'est à la juridiction qu'il appartient maintenant de juger si elle a respecté le droit ou non. Cette procédure est salutaire pour la démocratie et l'Etat de droit. Elle est pareillement bénéfique pour la politique européenne comme pour la cohésion de l'Union européenne.

(Traduction : I. Bourgeois)

\section{Indications bibliographiques}

- BANQUe CENTRALE eUROPÉENNE, Décision du Conseil des gouverneurs de la BCE du 6 septembre 2012, "Technical Features of Outright Monetary Transactions » (http://www.ecb.europa.eu/ press/ $\mathrm{pr} / \mathrm{date} / 2012 / \mathrm{html} / \mathrm{pr} 120906$ 1.en.html). Ce communiqué de presse se réfère à la $340^{\mathrm{e}}$ séance du Conseil des gouverneurs (05/06-09-2012) à Francfort ; dans son arrêt, la Cour de Karlsruhe cite de nombreux passage du compte rendu de cette séance (il n'est pas accessible au public)

- BundeSVERFASSUNGSGERICHT. Le communiqué de presse n9/2014 du 07-02-2014, également disponible en langue anglaise, rappelle toutes les références utiles: décisions et arrêts $2 \mathrm{BvR}$ 2728/13, 2 BvR 2729/13, 2 BvR 2730/13, 2 BvR 2731/13, 2 BvE 13/13; renvoi par le TCF à la CJUE : décisions du 17-12-2013 et du 14-01-2014 (www.bundesverfassungsgericht.de)

-Hallstein W., Die Europäische Gemeinschaft, $5^{e}$ réédition, Berlin, 1979

- MüLleR-Graff P.-C., «Das EuGH-Urteil zum Europäischen Stabilitätsmechanismus. Die itio in partes in der Europäischen Wirtschaftsunion : EU und ESM », RIW, 2013

-MüLLER-GrafF P.-C., «Die rechtliche Neujustierung der Europäischen Wirtschafts- und Währungsunion 》, ZHR 176/2012

- MüLLER-GRAFF P.-C., "L'arrêt de Karlsruhe à propos du MES ", "L'arrêt de Karlsruhe sur les aides budgétaires dans la zone Euro ", et "L'arrêt de Karlsruhe sur le Traité de Lisbonne ", Regards sur l'économie allemande, $\mathrm{n}^{\circ}$ 106/2012, 102/2011 et 92/2009

- von ARNIM H. H., Gemeinwohl und Gruppeninteressen, Francfort/Main, 1977. 